\title{
Existence of hyperons in the pulsar PSRJ1614-2230
}

\author{
A. Sulaksono ${ }^{1}$ and B. K. Agrawal ${ }^{2}$, \\ 1 Departemen Fisika, FMIPA, Universitas Indonesia, Depok, 16424, Indonesia. \\ ${ }^{2}$ Saha Institute of Nuclear Physics, Kolkata - 700064, India.
}

\begin{abstract}
The possibility of existence of hyperons in the recently measured $2 M_{\odot}$ pulsar PSRJ1614-2230 is explored using a diverse set of nuclear equations of state calculated within the relativistic meanfield models. Our results indicate that the nuclear equations of state compatible with heavy-ion data allow the hyperons to exist in the PSRJ1614-2230 only for significantly larger values for the meson-hyperon coupling strengths. The maximum mass configurations for these cases contain sizable hyperon fractions $(\sim 60 \%)$ and yet masquared their counterpart composed of only nucleonic matter.
\end{abstract}

PACS numbers: 21.30.Fe, 21.65.Cd, 26.60.-c 


\section{INTRODUCTION}

The latest measurement of the Shapiro delay for the millisecond pulsar PSRJ1614-2230 provides reliable lower bound on the maximum mass to be $1.97 \pm 0.04 M_{\odot}[1]$. This measurement rules out all the equations of state (EOSs) yielding the maximum mass less than that of the PSRJ1614-2230. Of course, the EOSs for the nucleonic matter can readily yield the compact stars with masses $\sim 2 M_{\odot}$. The EOSs with hadron-quark phase transition are also compatible with the mass measurement of the PSRJ1614-2230, provided, the quarks are assumed to be strongly interacting and are in colour superconducting phase [2, 3]. However, at large, the maximum mass of the compact stars are found to be well below $2 M_{\odot}$ when the non-nucleonic degrees of freedom like hyperons and kaon condensates are considered [4 9]. One might thus infer in the backdrop of previous calculations that the existence of hyperons and kaon condensates are unlikely in the PSRJ1614-2230.

Recently, studies involving role of hyperons on the maximum mass of the compact stars are revisited [10 14]. It is shown that the EOS for the hyperonic matter at higher densities can be stiffened in several ways within the relativistic mean-field (RMF) models. The maximum mass for these EOSs are above $2 M_{\odot}$ indicating the existence of hyperons in the PSRJ1614-2230. The EOS of the hyperonic matter are stiffened by including the vectorisoscalar strange $\phi$ mesons within the RMF model, in addition to the usual scalar-isoscalar $\sigma$, vector-isoscalar $\omega$ and vector-isovector $\rho$ mesons [13]. The $\phi$ mesons stiffens the EOS at densities far beyond the nuclear saturation density. Further, the increase of about $0.2 M_{\odot}$ in the maximum mass of the compact stars is achieved by varying the hyperon-nucleon potential depths for the $\Sigma$ and $\Xi$ hyperons which are not very well known. It has been also demonstrated [15] that maximum mass of the compact stars containing hyperons are well above $2 M_{\odot}$, provided, the $\omega$ mesons are coupled to hyperons as strongly as to the nucleons. Such strong meson-hyperon couplings stemmed from the breaking of SU(6) symmetry. Another way to stiffen the EOS is to include the non-linear terms for the $\phi$-mesons as suggested in Ref. [11]. It may be pointed out that the nuclear EOSs employed in Ref. [13, [15] are generally quite stiff in comparison to those extracted from the heavy-ion data [16 19].

An alternative scenario has also emerged in which compact stars containing hyperons can have masses $\sim 2 M_{\odot}$. In this scenario, one assumes the presence of hypothetical weakly interacting light bosons (WILBs) at densities several times of the nuclear saturation density. 
The role of WILBs on the EOS or the internal structure of the compact stars crucially depends on the choice of the characteristic scale which is the square of the ratio of the coupling strength to the masses of WILBs. The values of the characteristic scale of the WILBs are poorly constrained at present by the laboratory data and the compact star observable [20 23]. Consequently, the nuclear EOSs compatible with the heavy-ion data allow the hyperons to exist in the compact stars with mass $\sim 2 M_{\odot}[24]$. The required stiffness of the EOS at very high density is achieved by adjusting the characteristic scale of the WILBs.

In the present work we explore the possibility of existence of hyperons in the PSRJ16142230 using a diverse set of nuclear EOSs calculated within the RMF models. In particular, we examine whether the nuclear EOSs compatible with the heavy-ion data can allow the hyperons to exist in the PSRJ1614-2230 without recourse to the WILBs. This makes our present investigation quite different than the ones performed earlier. Most of the previous investigations either employed the nuclear EOSs which are stiffer in comparison to the heavy-ion data or included the contributions of the WILBs.

The paper is organized as follows. In Sec. II we describe the theoretical framework based on the RMF model. In Sec. III, we present our results for the EOSs for the matter without and with hyperons. In Sec. IV we present our results for the bulk properties of the static compact stars. Finally, we state our conclusions in Sec. V.

\section{THEORETICAL FRAME WORK}

We use standard and extended versions of the RMF models to compute the bulk properties of the compact stars. The standard RMF model includes the contributions from the nonlinear self-interaction for the $\sigma$ meson and extended RMF model includes the contributions from the self and/or cross interaction terms for the $\sigma, \omega$ and $\rho$ meson. We will discuss both versions of the RMF models. The derivations of the effective Lagrangian density and corresponding energy density functionals for the extended RMF model are well documented in Refs. [25 27]. In RMF models, baryons interact through the exchange of scalar $(\sigma)$, vector ( $\omega$ and $\rho$ ) as well as two additional hidden-strangeness $\left(\sigma^{*}\right.$ and $\left.\phi\right)$ mesons. The baryons considered in this work are nucleon $(N)$ and hyperons $(\Lambda, \Sigma, \Xi)$. The total Lagrangian density of RMF model including hyperons plus leptons (l) can be written as, 


$$
\mathcal{L}=\mathcal{L}_{B}^{\text {free }}+\mathcal{L}_{M}^{\text {free }}+\mathcal{L}_{B M}^{\text {lin }}+\mathcal{L}^{\text {nonlin }}+\mathcal{L}_{l}^{\text {free }}
$$

where the free baryons Lagrangian density is,

$$
\mathcal{L}_{B}^{\text {free }}=\sum_{B=N, \Lambda, \Sigma, \Xi} \bar{\Psi}_{B}\left[i \gamma^{\mu} \partial_{\mu}-M_{B}\right] \Psi_{B}
$$

Here, $\Psi_{B}$ is baryons field and the sum is taken over $N, \Lambda, \Sigma$,and $\Xi$ baryons. The Lagrangian density for free mesons involved is,

$$
\begin{aligned}
\mathcal{L}_{M}^{\text {free }} & =\frac{1}{2}\left(\partial_{\mu} \sigma \partial^{\mu} \sigma-m_{\sigma}^{2} \sigma^{2}\right)+\frac{1}{2}\left(\partial_{\mu} \sigma^{*} \partial^{\mu} \sigma^{*}-m_{\sigma^{*}}^{2} \sigma^{* 2}\right) \\
& -\frac{1}{4} \omega_{\mu \nu} \omega^{\mu \nu}+\frac{1}{2} m_{\omega}^{2} \omega_{\mu} \omega^{\mu}-\frac{1}{4} \phi_{\mu \nu} \phi^{\mu \nu}+\frac{1}{2} m_{\phi}^{2} \phi_{\mu} \phi^{\mu} \\
& -\frac{1}{4} \rho_{\mu \nu} \rho^{\mu \nu}+\frac{1}{2} m_{\rho}^{2} \rho_{\mu} \rho^{\mu} .
\end{aligned}
$$

The $\omega^{\mu \nu}, \phi^{\mu \nu}$ and $\rho^{\mu \nu}$ are field tensors corresponding to the $\omega, \phi$ and $\rho$ mesons field, and can be defined as $\omega^{\mu \nu}=\partial^{\mu} \omega^{\nu}-\partial^{\nu} \omega^{\mu}, \phi^{\mu \nu}=\partial^{\mu} \phi^{\nu}-\partial^{\nu} \phi^{\mu}$ and $\rho^{\mu \nu}=\partial^{\mu} \rho^{\nu}-\partial^{\nu} \rho^{\mu}$. The Lagrangian $\mathcal{L}_{B M}^{\operatorname{lin}}$ describing the interactions of the baryons through the mesons is,

$$
\mathcal{L}_{B M}^{\operatorname{lin}}=\sum_{B=N, \Lambda, \Sigma, \Xi} \bar{\Psi}_{B}\left[g_{\sigma B} \sigma+g_{\sigma^{*} B} \sigma^{*}-\gamma_{\mu} g_{\omega B} \omega^{\mu}-\frac{1}{2} \gamma_{\mu} g_{\rho B} \tau_{\mathbf{B}} \cdot \rho^{\mu}-\gamma_{\mu} g_{\phi B} \phi^{\mu}\right] \Psi_{B}
$$

where $\tau_{B}$ are the baryons isospin matrices. The Lagrangian describing nucleons self interactions for $\sigma, \omega$, and $\rho$ mesons can be written as,

$$
\begin{aligned}
\mathcal{L}^{\text {nonlin }} & =-\frac{\kappa_{3} g_{\sigma N} m_{\sigma}^{2}}{6 m_{N}} \sigma^{3}-\frac{\kappa_{4} g_{\sigma N}^{2} m_{\sigma}^{2}}{24 m_{N}^{2}} \sigma^{4}+\frac{\zeta_{0} g_{\omega N}^{2}}{24}\left(\omega_{\mu} \omega^{\mu}\right)^{2} \\
& +\frac{\eta_{1} g_{\sigma N} m_{\omega}^{2}}{2 m_{N}} \sigma \omega_{\mu} \omega^{\mu}+\frac{\eta_{2} g_{\sigma N}^{2} m_{\omega}^{2}}{4 m_{N}^{2}} \sigma^{2} \omega_{\mu} \omega^{\mu} \\
& +\frac{\eta_{\rho} g_{\sigma N} m_{\rho}^{2}}{2 m_{B}} \sigma \rho_{\mu} \cdot \rho^{\mu}+\frac{\eta_{1 \rho} g_{\sigma N}^{2} m_{\rho}^{2}}{4 m_{N}^{2}} \sigma^{2} \rho_{\mu} \cdot \rho^{\mu}+\frac{\eta_{2 \rho} g_{\omega N}^{2} m_{\rho}^{2}}{4 m_{N}^{2}} \omega_{\mu} \omega^{\mu} \rho_{\mu} \cdot \rho^{\mu}
\end{aligned}
$$

While the free leptons Lagrangian density is,

$$
\mathcal{L}_{l}^{\text {free }}=\sum_{l=e^{-}, \mu^{-}} \bar{\Psi}_{l}\left[i \gamma^{\mu} \partial_{\mu}-M_{l}\right] \Psi_{l}
$$

here $\Psi_{l}$ is leptons (electron and muon) field.

The meson-nucleon coupling constants and nonlinear parameters of RMF models are determined by adjusting them to reproduce ground state properties of finite nuclei and nuclear matter. Here, we employed NL3, GM1, TM1, G2, BSR2, BSR6, BSR9, BSR13 and 
BSP parameter sets for meson-nucleon coupling constants and nonlinear parameters [4, 2730]. These parameterizations of the RMF models are associated with different form for the Lagrangian density. They differ in their non-linear part of the effective Lagrangian (Eq. 5) which are summarized in Table I. For instance, the Lagrangian density associated with the parameter sets NL3 and GM1 correspond to the standard RMF model which includes non-linear term only for the self-interactions of the $\sigma$ meson. Other parameter sets TM1, G2 and BSR correspond to the extended RMF models which include contributions from the self-interactions of the $\omega$ mesons and/or cross-interactions between $\sigma, \omega$ and $\rho$ mesons.

The meson-hyperon coupling strengths $g_{m H}\left(m=\sigma, \omega, \rho, \sigma^{*}, \phi\right.$ and $\left.H=\Lambda, \Sigma, \Xi\right)$ in Eq. (4) can not be determined very well at present. The properties of compact stars are quite sensitive to the choices for the values of $g_{\sigma H}, g_{\omega H}$ and $g_{\phi H}$. The variations in the values of $g_{\rho H}$ and $g_{\sigma^{*} H}$ do not appreciably alter the properties of compact stars. The values of $g_{\rho H}$ and $g_{\sigma^{*} H}$ are kept fixed in our calculations. We take $g_{\sigma^{*} H}=0$, this choice is consistent with $\Lambda \Lambda$-hypernuclear data which yields weak $\Lambda \Lambda$ interaction [31]. The values of $g_{\rho H}$ are taken from the SU(6) quark model. We make several choices for the values of $g_{\omega H}$ and $g_{\phi H}$. One of which correspond to those obtained within the SU(6) quark model. The SU(6) quark model values for $g_{\omega H}, g_{\rho H}$ and $g_{\phi H}$ are

$$
\begin{aligned}
\frac{1}{3} g_{\omega N} & =\frac{1}{2} g_{\omega \Lambda}=\frac{1}{2} g_{\omega \Sigma}=g_{\omega \Xi}, \\
g_{\rho N} & =\frac{1}{2} g_{\rho \Sigma}=g_{\rho \Xi}, \quad g_{\rho \Lambda}=0, \\
2 g_{\phi \Lambda} & =2 g_{\phi \Sigma}=g_{\phi \Xi}=\frac{2 \sqrt{2}}{3} g_{\omega N}, \quad g_{\phi N}=0 .
\end{aligned}
$$

For given values of $g_{\omega H}$ the coupling strengths $g_{\sigma H}$ are usually obtained from potential depths for hyperons in the symmetric nuclear matter at the saturation density as,

$$
U_{H}^{(N)}\left(\rho_{s}\right)=-g_{\sigma H} \sigma\left(\rho_{s}\right)+g_{\omega H} \omega\left(\rho_{s}\right)
$$

The values of potential depths $U_{H}^{(N)}$ chosen are as follows [32],

$$
U_{\Lambda}^{(N)}=-28 \mathrm{MeV}, \quad U_{\Sigma}^{(N)}=+30 \mathrm{MeV} \quad \text { and } \quad U_{\Xi}^{(N)}=-18 \mathrm{MeV} .
$$

For the sake of convenience we define, 


$$
X_{\omega H}=\left\{\begin{array}{lc}
\left(\frac{g_{\omega H}}{g_{\omega N}}\right) & \text { for } \Lambda \text { and } \Sigma \text { hyperons } \\
2\left(\frac{g_{\omega H}}{g_{\omega N}}\right) & \text { for } \Xi \text { hyperons }
\end{array}\right.
$$

and,

$$
X_{\phi H}=\left\{\begin{array}{cc}
\left(\frac{g_{\phi H}}{g_{\omega N}}\right) & \text { for } \Lambda \text { and } \Sigma \text { hyperons } \\
\frac{1}{2}\left(\frac{g_{\phi H}}{g_{\omega N}}\right) & \text { for } \Xi \text { hyperons. }
\end{array}\right.
$$

It has been shown earlier in Ref. [4] that the values of $X_{\omega H} \approx 1$, which are larger compared to those for the SU(6) model (Eq. 7), can yield heavier compact stars composed of hyperons without affecting the properties of the $\Lambda$-hypernuclei. Recent investigation [15] suggests that the values of $X_{\omega H}$ can significantly differ from those given by the $\mathrm{SU}(6)$ quark model. The breaking of the $\mathrm{SU}(6)$ symmetry yields $X_{\omega \Lambda}=X_{\omega \Sigma}=\frac{1}{2} X_{\omega \Xi}=1$ (i.e.,

$\left.g_{\omega \Lambda}=g_{\omega \Sigma}=g_{\omega \Xi}=g_{\omega N}\right)$. In the present work we vary the values of $X_{\omega H}$ and $X_{\phi H}$ over a wide range to see in what limits the compact stars with hyperons can satisfy the constraints on the lower bound on the maximum mass imposed by the PSRJ1614-2230.

\section{EQUATIONS OF STATE}

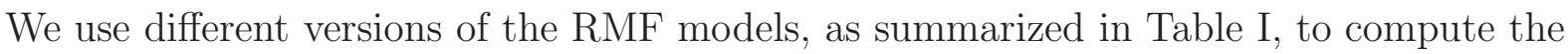
EOSs for the $\beta$-equilibrated nucleonic and hyperonic matters. Our choice for the models are such that they yield wide variations in the various quantities associated with symmetric and asymmetric nuclear matter at the saturation density. In Table II we provide the values of some of the quantities associated with nuclear matter at the saturation density $\rho_{s}$, namely, the binding energy per nucleons $(B / A)$, incompressibility coefficient for symmetric nuclear matter $(K)$, symmetry energy coefficient $\left(E_{\text {sym }}\right)$, linear density dependence of the symmetry energy coefficient $(L)$ and various quantities $\left(K_{\text {sym }}\right),\left(K_{\text {asy }}\right)$ and $\left(K_{\text {sat } 2}\right)$. These quantities are evaluated as follows, 


$$
\begin{array}{r}
E_{\mathrm{sym}}(\rho)=\left.\frac{1}{2} \frac{d^{2} E(\rho, \delta)}{d \delta^{2}}\right|_{\delta=0}, \\
L=\left.3 \rho_{s} \frac{d E_{\mathrm{sym}}(\rho)}{d \rho}\right|_{\rho=\rho_{s}}, \\
K_{\mathrm{sym}}=\left.9 \rho_{s}^{2} \frac{d^{2} E_{\mathrm{sym}}(\rho)}{d \rho^{2}}\right|_{\rho=\rho_{s}}, \\
K=\left.9 \rho_{s}^{2} \frac{d^{2} E(\rho, 0)}{d \rho^{2}}\right|_{\rho=\rho_{s}}, \\
J=\left.27 \rho_{s}^{3} \frac{d^{3} E(\rho, 0)}{d \rho^{3}}\right|_{\rho=\rho_{s}}, \\
K_{\mathrm{sat}, 2}=K_{\mathrm{asy}}-\frac{J}{K} L, \\
K_{\mathrm{asy}}=K_{\mathrm{sym}}-6 L .
\end{array}
$$

where, $E(\rho, \delta)$ is the energy per nucleon at a given density $\rho$ and asymmetry $\delta=\left(\rho_{n}-\rho_{p}\right) / \rho$. In Table I, we also list the values of the neutron-skin $\Delta R$ for the ${ }^{208} \mathrm{~Pb}$ nucleus. The values of $K, E_{\text {sym }}$ and $L$ vary over a wide range for the selected RMF model. This immediately indicates that the behaviour of the EOSs for the different RMF models considered should be quite different even at the low densities.

In Fig. 1 we plot the EOSs for symmetric nuclear matter (SNM) in terms of pressure versus nucleon density obtained for various RMF models. The bounds on the EOSs as shown by shaded regions are the ones extracted from heavy-ion collision data [19]. The EOSs for the NL3, GM1, BSR2 and BSR6 are stiffer in comparison to those obtained from the heavy-ion data. Whereas, the TM1, G2, BSR9, BSR13 and BSP are consistent with the EOS from the heavy-ion data. The EOS for the BSR2 and BSR6 parameterizations are very much the same for the reasons as follow. Both of the parameter sets belong to the same type of the RMF model as can be seen from Table I. Further, the parameters of the BSR2 and BSR6 parameterizations are obtained by fit to exactly same set of experimental data for bulk properties of finite nuclei, but, for different values of the neutron-skin thickness in ${ }^{208} \mathrm{~Pb}$ nucleus. Thus, the BSR2 and BSR6 parameterizations are expected to yield different behaviour only for the EOSs of the asymmetric nuclear matter. Similar is the case for the EOSs obtained with the BSR9 and BSR13 parameterizations. 
Our main goal is to investigate whether the nuclear EOSs which are compatible with the heavy-ion data can allow the hyperons to exist in the $2 M_{\odot}$ pulsar PSRJ1614-2230. For this purpose, the EOSs for the $\beta$-equilibrated hyperonic matter are calculated using different values for $X_{\omega H}$ and $X_{\phi H}$. We present here the EOSs only for two different choices for the $X_{\omega H}$ and $X_{\phi H}$. We refer these choices as XSU6 and X180. For the case of XSU6, $X_{\omega H}=\frac{2}{3}$ and $X_{\phi H}=\frac{\sqrt{2}}{3}$ which correspond to those given by the $\mathrm{SU}(6)$ quark model as conventionally used. The X180 refers to $X_{\omega H}=1$ and $X_{\phi H}=0.8$. The values of $\sigma-H$ coupling strength are determined using the nucleon-hyperon potential depths as described in Sec. II. It can be easily verified from Eqs. (7, 10 and 11) that the values of $X_{\omega H}$ and $X_{\phi H}$ for the case of X180 are augmented by a factor of $\sim 1.5$ with respect to their values for the $\mathrm{SU}(6)$ model. In Fig. 2, the EOSs for the nucleonic and hyperonic matters are compared for a few different RMF models. The EOSs for the nucleonic matter are labeled as 'NO HYP'. The EOSs for the hyperonic matter are labeled as XSU6 and X180. It can be seen that the EOSs for the hyperonic matter corresponding to X180 are quite close to those obtained for the nucleonic matter. It should be pointed out that the threshold density is lowest for the $\Lambda$ hyperons for all the RMF models considered irrespective of the choice of the meson-hyperon coupling strengths. Further, the augmented meson-hyperon coupling strengths results in marginal increase in the values of the threshold densities for the $\Lambda$ hyperons. For instance, the threshold densities for $\Lambda$ hyperons for the NL3(BSP) parameterizations for the XSU6 and $\mathrm{X} 180$ cases are $0.28(0.38)$ and $0.32(0.42) \mathrm{fm}^{-3}$,respectively. Thus, the augmented mesonhyperon coupling strengths might increases the maximum mass of the compact stars without significantly affecting its hyperonic contents.

Before employing our EOSs for the hyperonic matter to study the properties of the compact stars, we would like to look into the behaviour of the $\Lambda-\mathrm{H}$ potentials $U_{H}^{(\Lambda)}$. The values of $\Lambda-\mathrm{H}$ for given meson-hyperon coupling strengths are obtained as,

$$
U_{H}^{(\Lambda)}(\rho)=-g_{\sigma H} \sigma(\rho)+g_{\omega H} \omega(\rho)+g_{\phi H} \phi(\rho)
$$

where, the values of the fields $\sigma, \omega$ and $\phi$ are calculated for the pure $\Lambda$ matter at a given density $\rho$. In Fig. 3, we plot the results for the $U_{H}^{(\Lambda)}$ obtained using the meson-hyperon coupling strengths corresponding to XSU6 and X180. For the comparison, we also plot the values of $U_{H}^{(\Lambda)}$ obtained for two different models based on the $\mathrm{SU}(3)$ symmetry [15, 33]. The green dashed curve is obtained using Ref. [15] for $Z=0$ which yields highest value for the 
maximum mass for the compact star. The values of $U_{\Lambda}^{(\Lambda)}$ and $U_{\Sigma}^{(\Lambda)}$ for the case of X180 lie within those obtained using other models. Whereas, the potential $U_{\Xi}^{(\Lambda)}$ is somewhat stiffer in comparison to the other models as considered.

\section{COMPACT STARS}

The properties of static or non-rotating compact stars for a given EOS is obtained by solving the Tolman-Oppenheimer-Volkoff (TOV) equations [34]. For the case of rotating compact stars we solve the Einstein equations for stationary axi-symmetric spacetime. The numerical computations are performed using the code written by Stergioulas [35]. We describe the outer crust region of the compact star using the EOS of Rüster et al. [36] which is the recent update of the one given by Baym, Pethick, and Sutherland [37]. Due to the fact that the detailed EOS of inner crust indeed is not yet certain, the polytropic pressure-energy density relation is used to interpolate the EOS for the region between outer crust and the core [38]. The core is assumed to be composed of either the nucleonic or the hyperonic matter in $\beta$-equilibrium. The EOS of the core is obtained from the different parameter sets of the RMF models as presented in Sec. III.

In Fig. 4 we display our results for the mass-radius relationships for the static compact stars composed of $\beta$-equilibrated nucleonic matter (upper panel) and hyperonic matter (lower panel). The shaded region represents the current lower limit on the maximum mass, $M=$ $1.97 \pm 0.04 M_{\odot}$, of the compact star obtained from the recent mass measurement of the PSRJ1614-2230. The mass-radius relationships for the compact stars containing hyperons are obtained using standard values for the meson-hyperon coupling strengths as discussed in the previous section. It is evident from the upper panel that the maximum masses of the compact star in the absence of hyperons for all the RMF models considered are compatible with the current limit on its lower bound. The solid circles in the lower panel indicate the minimum mass of the compact star at which the hyperons begin to appear. This minimum mass ranges from $1.2-1.5 M_{\odot}$ for the various RMF models considered. The parameter sets NL3, GM1, BSR2 and BSR6 yield maximum mass larger than $2 M_{\odot}$ when the hyperons are included. Thus, these parameter sets readily allow the hyperons to appear in the PSRJ16142230. The nuclear EOSs, however, for these cases are not compatible with the heavy-ion data (see Fig. 1). On the other hand, the nuclear EOSs for the parameter sets BSR9, BSR13, 
BSP, G2 and TM1 are compatible with the heavy-ion data, but, they yield the maximum mass in the range of $1.7-1.9 M_{\odot}$ with the inclusion of hyperons. It seems that the nuclear EOSs, compatible with heavy-ion data, can possibly allow the hyperons to exist in the PSRJ1614-2230 for substantially larger values of the meson-hyperon coupling strengths in comparison to their standard values.

In Fig. 5 we show the mass-radius relationships for the compact stars obtained by augmenting the meson-hyperon coupling strengths in comparison to their standard values. As discussed previously, the label X180 corresponds to $X_{\omega H}=1$ and $X_{\phi H}=0.8$ which is larger by a factor of $\sim 1.5$ with respect to their slandered values taken from the $\mathrm{SU}(6)$ quark model. We plot the results only for those RMF model for which the nuclear EOSs are compatible with the heavy-ion data. We find that the maximum masses for the TM1 and BSP parameter sets are consistent with the mass of the PSRJ1614-2230. It may be pointed out to this end that the radius $R_{1.4}$ for the compact star with canonical mass of $1.4 M_{\odot}$ for the BSP case is $12.7 \mathrm{~km}$ which is consistent with $R_{1.4}=10.4-12.9 \mathrm{~km}$ as determined from the recent observations of both transiently accreting and bursting sources [39]. The values of $R_{1.4}$ determined in Ref. [39] are independent of assumptions about the composition of the core. We also look into the hyperon fractions at the maximum mass. In Table III we present our results for the maximum mass configurations obtained for two different choices of meson-hyperon coupling strengths corresponding to XSU6 and X180. These results are compared with their nuclear counterparts having no hyperons. We also list the values of the partial mass $M_{\max }^{\mathrm{HYP}}$ which is composed of the hyperonic matter. The values of $M_{\max }^{\text {HYP }}$ are obtained by integrating the density profile, corresponding to the maximum mass configuration, from the central density to the threshold density at which hyperons begin to appear. It is interesting to note that the ratio $M_{\max }^{\mathrm{HYP}} / M_{\max }$ is sizable and almost the same for both the choices of the meson-hyperon coupling strengths. The maximum mass obtained using augmented meson-hyperon coupling strengths are very close to their nuclear counter parts and yet significant fraction $(\sim 60 \%)$ of the mass is composed of the hyperonic matter. Further, the radius $R_{\max }$ at the maximum mass for the case with no hyperons and the one obtained using augmented meson-hyperon coupling strengths are almost the same. Thus, it seems possible to obtain the maximum mass configuration of the compact star, containing sizable fraction of hyperons, which masquareds its nuclear counterpart. The maximum mass of the compact stars with hyperons nearly same as their nuclear counterpart has also been 
found in Ref. [15, 33]

In Fig. 6] we plot the variations of $M_{\max }$ with $X_{\omega H}$ and $X_{\phi H}$ for the case of BSP. The value of $M_{\max }$ increases with increasing $X_{\omega H}$ and $X_{\phi H}$. The $M_{\max }$ depends strongly on $X_{\omega H}$ for smaller values of $X_{\phi H}$. As the $X_{\phi H}$ increases, the value of $M_{\max }$ tends to saturate and becomes more or less independent of $X_{\omega H}$. For $X_{\phi H}=1$, the $M_{\max }$ increases by $0.1 M_{\odot}$ with increase in $X_{\omega H}$ from 0.5 to 1 . Also, the $M_{\max }$ is less dependent on $X_{\phi H}$ for larger $X_{\omega H}$. To this end, we would like to remind that the $\Sigma-N$ potential depth $U_{\Sigma}^{(N)}$ is taken to be $30 \mathrm{MeV}$. The value of $U_{\Sigma}^{(N)}$ is not yet certain. In Fig. 7, we compare our results for the $M_{\max }$ obtained for $U_{\Sigma}^{(N)}=-30$ and $30 \mathrm{MeV}$. The value of $M_{\max }$ depends somewhat on $U_{\Sigma}^{(N)}$ for smaller values of $X_{\Omega H}$. For $X_{\Omega H}=0.5$, the $M_{\max }$ may increases by $\sim 0.1 M_{\odot}$ with increase in $U_{\Sigma}^{(N)}$ from -30 to $30 \mathrm{MeV}$. We also calculate the maximum mass of the compact stars rotating with the frequency $\nu=317 \mathrm{~Hz}$ as that of the PSRJ1614-2230. In Fig. 8, we display the variations for the $M_{\max }$ with $X_{\omega H}$ and $X_{\phi H}$. It can be easily seen that the value of $X_{\omega H}=1$ with $X_{\phi H}=0.65$ is sufficient to produce the compact star with mass compatible with the current lower bound on the $M_{\max }$.

\section{CONCLUSIONS}

We use various RMF models to explore the possibility of existence of hyperons in the heaviest observed compact star PSRJ1614-2230 having mass $M=1.97 \pm 0.04 M_{\odot}$. We have examined the conditions required for the hyperons to exist in the PSRJ1614-2230 when the nuclear EOSs are subjected to the constrained imposed by the the heavy-ion data. The various RMF models are selected in such a way that they result in a diverse set of nuclear EOSs. The values of maximum mass for these models, in the absence of hyperons, are consistent with the mass of PSRJ1614-2230. The nuclear EOSs which are consistent with the heavy-ion data require larger values of the meson-hyperon coupling strengths in order to allow the hyperons to exist in the PSRJ1614-2230. Particularly, the coupling of $\omega$ and $\phi$ vector mesons to the hyperons are required to be significantly augmented with respect to their standard values. These lager values of the coupling strengths increases the maximum mass to the desired limit without significantly affecting the fraction of maximum mass composed of hyperonic matter. We find that the maximum mass configuration of the compact star with sizable fraction of hyperons masquareds its nuclear counterpart. 
[1] P. B. Demorest, T. Pennucci, S. M. Ransom, M. S. E. Roberts, and J. W. T. Hessels, Nature 467, 1081 (2010).

[2] F. Ozel, D. Psaltis, S. Ransom, P.Demorest, and M. Alford, Astrophys. J.Lett. 724, L199 (2010).

[3] S. Weissenborn, I.Sagret, G.Pagliara, M.Hempel, and J. Schaffnre-Bielich, Astrophys. J. Lett. 740, L14 (2011).

[4] N. K. Glendenning and S. A. Moszkowski, Phys. Rev. Lett. 67, 2414 (1991).

[5] N. K. Glendenning and J. Schaffner-Bielich, Phys. Rev. Lett. 81, 4564 (1998).

[6] B. D. Lackey, M. Nayyar, and B. Owen, Phys. Rev. D 73, 024021 (2006).

[7] H. J. Schulze, A. Polls, A. Ramos, and I. Vidana, Phys. Rev. C 73, 058801 (2006).

[8] J. M. Lattimer and M. Prakesh, Phys. Rep. 442, 109 (2007).

[9] I. Bednarek and R. Manka, Eur. Phys. Lett. 78, 32001 (2007).

[10] J. R. Stone, P. Guichon, and A.W.Thomas, arxiv:1012.2919v1 (2010).

[11] I. Bednarek, P. Haensel, J. L. Zdunik, M. Bejger, and R. Manka, Astron. Astrophys. 543, A157 (2012).

[12] J. E. Trumper, Prog. Part. Nucl Phys. 66, 674 (2011).

[13] S. Weissenborn, D. Chatterjee, and J. Schaffnre-Bielich, Nucl. Phys. A 881, 62 (2012).

[14] D. Logoteta, I. Bombaci, C. Providencia, and I. Vidana, Phys. Rev. D 85, 023003 (2012).

[15] S. Weissenborn, D. Chatterjee, and J. Schaffnre-Bielich, Phys. Rev. C 85, 065802 (2012).

[16] J. Aichelin and C. M. Ko, Phys. Rev. Lett. 55, 2661 (1985).

[17] C. Fuchs, A. Faessler, and E. Zabrodin, Phys. Rev. Lett. 86, 1974 (2001).

[18] C. Fuchs, J. Phys. G 35, 14049 (2008).

[19] P. Danielewicz, W. G. Lynch, and R. Lacey, Science 298, 1592 (2002).

[20] S. K. Lamoreaux, Phys. Rev. Lett. 78, 5 (1997).

[21] R. S. Decca, D. Lopez, H. B. Chan, E. Fischbach, D. E. Krause, and C. R. Jamell, Phys. Rev. Lett. 94, 240401 (2005).

[22] V. M. Mostepanenko, R. S. Decca, E. Fischbach, G. L. Klimchitskaya, D. E. Krause, and D. Lopez, J. Phys. A 41, 164054 (2008).

[23] M. I. Krivoruchenko, F.Simkovic, and A. Faessler, Phys. Rev. D 79, 125023 (2009). 
[24] A. Sulaksono, Marliana, and Kasmudin, Mod. Phys. Lett. A 26, 367 (2011).

[25] R. Furnstahl, B. D. Serot, and H.-B. Tang, Nucl. Phys. A598, 539 (1996).

[26] B. D. Serot and J. D. Walecka, Int. J. Mod. Phys. E 6, 515 (1997).

[27] R. Furnstahl, B. D. Serot, and H.-B. Tang, Nucl. Phys. A615, 441 (1997).

[28] G. A. Lalazissis, J. Konig, and P. Ring, Phys. Rev. C 55, 540 (1997).

[29] S. K. Dhiman, R. Kumar, and B. K. Agrawal, Phys. Rev. C 76, 045801 (2007).

[30] B. K. Agrawal, Phys. Rev. C 81, 034323 (2010).

[31] A. Gal and D. Millener, Phys. Lett. B701, 342 (2011).

[32] J. Schaffner-Bielich and A. Gal, Phys. Rev. C 62, 034311 (2000).

[33] K. Tsubakihara, H. Maekawa, H. Matsumiya, and A. Ohnishi, Phys. Rev. C 81, 065206 (2010).

[34] S. Weinberg, Gravitation and Cosmology (Wiley, New York, 1972).

[35] N. Stergioulas and J. L. Friedman, Astrophys. J. 444, 306 (1995).

[36] S. B. Ruster, M. Hampel, and J. Schaffner-Bielich, Phys. Rev. C 73, 035804 (2006).

[37] G. Baym, C. Pethick, and P. Sutherland, Astrophys. J. 170, 299 (1971).

[38] F.J.Fattoyev, C.J.Horowitz, J. Piekarewicz, and G.Shen, Phys. Rev. C 82, 055803 (2010).

[39] A. W. Steiner, J. M. Lattimer, and E. F. Brown, arxiv:1205.26871 (2012).

[40] B. K. Agrawal, A. Sulaksono, and P. G. Reinhard, Nucl. Phys. A 882, 1 (2012).

[41] Y.Sugahara and H.Toki, Nucl. Phys. A579, 557 (1994). 
TABLE I: Various self-interaction and cross-interaction terms included in the Lagrangian density associated with different parameterizations of the RMF models considered in the present work. The index '1' and ' 0 ' is used to indicate whether or not the corresponding term is included.

\begin{tabular}{|c|c|c|c|c|c|c|c|}
\hline \multirow[t]{2}{*}{ Parameter } & \multicolumn{3}{|c|}{ Self-interaction } & \multicolumn{3}{|c|}{ Cross-interaction } & \multirow[t]{2}{*}{ Ref. } \\
\hline & $\sigma$ & $\omega$ & $\rho$ & $\sigma-\omega$ & $\sigma-\rho$ & $\omega-\rho$ & \\
\hline BSP & 1 & 0 & 0 & 1 & 0 & 1 & [40] \\
\hline BSR13 & 1 & 1 & 0 & 1 & 1 & 1 & {$[29,30$} \\
\hline BSR9 & 1 & 1 & 0 & 1 & 1 & 1 & {$[29,30$} \\
\hline BSR6 & 1 & 0 & 0 & 1 & 1 & 1 & {$[29,30$} \\
\hline $\mathrm{BSR} 2$ & 1 & 0 & 0 & 1 & 1 & 1 & {$[29,30$} \\
\hline G2 & 1 & 1 & 0 & 1 & 1 & 0 & [27] \\
\hline TM1 & 1 & 1 & 0 & 0 & 0 & 0 & [41] \\
\hline GM1 & 1 & 0 & 0 & 0 & 0 & 0 & {$[4]$} \\
\hline NL3 & 1 & 0 & 0 & 0 & 0 & 0 & [28] \\
\hline
\end{tabular}


TABLE II: Some bulk properties of the nuclear matter at the saturation density $\left(\rho_{s}\right)$ : binding energy per nucleon $(B / A)$, incompressibility coefficient for symmetric nuclear matter $(K)$, symmetry energy $\left(E_{\text {sym }}\left(\rho_{s}\right)\right)$, linear density dependence of the symmetry energy $(L)$ and various quantities $\left(K_{\text {sym }}\right),\left(K_{\text {asy }}\right)$ and $\left(K_{\text {sat2 }}\right)$ as given by Eqs. (16 18). The values for the neutron-skin thickness $\Delta R$ for the ${ }^{208} \mathrm{~Pb}$ nucleus are also listed. The values of $\rho_{s}$ are in $\mathrm{fm}^{-3}, \Delta R$ in $\mathrm{fm}$ and all the other quantities are in $\mathrm{MeV}$.

\begin{tabular}{|cccccccccc|}
\hline \hline Parameter & $\Delta_{R}$ & $B / A$ & $\rho_{s}$ & $K$ & $E_{\text {sym }}\left(\rho_{s}\right)$ & $L$ & $K_{\text {sym }}$ & $K_{\text {asy }}$ & $K_{\text {sat2 }}$ \\
\hline BSP & 0.15 & 15.9 & 0.149 & 230 & 28.83 & 50 & 9 & -290 & -218 \\
BSR13 & 0.26 & 16.1 & 0.147 & 229 & 35.6 & 91 & -40 & -585 & -466 \\
BSR9 & 0.18 & 16.1 & 0.147 & 233 & 31.6 & 64 & -12 & -396 & -313 \\
BSR6 & 0.26 & 16.1 & 0.149 & 236 & 35.4 & 86 & -48 & -562 & -557 \\
BSR2 & 0.18 & 16.0 & 0.149 & 240 & 31.4 & 62 & -4 & -376 & -363 \\
G2 & 0.26 & 16.1 & 0.153 & 215 & 36.4 & 100 & -7 & -611 & -404 \\
GM1 & 0.23 & 16.3 & 0.153 & 300 & 32.5 & 94 & 18 & -545 & -466 \\
TM1 & 0.27 & 16.3 & 0.145 & 281 & 36.8 & 111 & 34 & -632 & -518 \\
NL3 & 0.28 & 16.3 & 0.148 & 272 & 37.4 & 118 & 100 & -608 & -700 \\
\hline \hline
\end{tabular}


TABLE III: The values of central density $\rho_{c}\left(\right.$ in $\left.\mathrm{fm}^{-3}\right)$, radius $R_{\max }$ (in $\mathrm{km}$ ) at the maximum mass $M_{\max }\left(\right.$ in $M_{\odot}$ ) for the cases with no hyperons and with hyperons. The quantity $M_{\max }^{\mathrm{HYP}}$ represents the partial mass composed of the hyperonic matter. The label XSU6 denotes the standard values for the meson-hyperon coupling strengths. The label X180 represents augmented values of the meson-hyperon coupling strengths.

\begin{tabular}{|c|c|c|c|c|c|c|c|c|c|c|c|c|c|}
\hline \multirow[t]{2}{*}{ Parameter } & \multicolumn{3}{|c|}{ NO HYP } & \multicolumn{5}{|c|}{ XSU6 } & \multicolumn{5}{|c|}{ X180 } \\
\hline & $\rho_{c}$ & $R_{\max }$ & $M_{\max }$ & $\rho_{c}$ & $R_{\max }$ & $M_{\max }$ & $M_{\max }^{\mathrm{HYP}}$ & $\frac{M_{\max }^{\mathrm{HYP}}}{M_{\max }}$ & $\rho_{c}$ & $R_{\max }$ & $M_{\max }$ & $M_{\max }^{\mathrm{HYP}}$ & $\frac{M_{\max }^{\mathrm{HYP}}}{M_{\max }}$ \\
\hline BSP & 1.04 & 10.91 & 2.00 & 1.12 & 10.83 & 1.73 & 1.14 & 0.66 & 1.08 & 10.98 & 1.96 & 1.29 & 0.66 \\
\hline BSR13 & 0.98 & 11.66 & 1.93 & 1.06 & 11.53 & 1.7 & 1.11 & 0.65 & 1.09 & 11.81 & 1.89 & 1.25 & 0.66 \\
\hline BSR9 & 1.00 & 11.36 & 1.92 & 1.07 & 11.26 & 1.7 & 1.07 & 0.63 & 1.02 & 11.44 & 1.88 & 1.16 & 0.60 \\
\hline BSR6 & 0.82 & 12.12 & 2.40 & 0.92 & 11.91 & 2.06 & 1.46 & 0.71 & 0.86 & 12.16 & 2.34 & 1.61 & 0.68 \\
\hline BSR2 & 0.85 & 11.84 & 2.35 & 0.94 & 11.65 & 2.03 & 1.4 & 0.69 & 0.87 & 11.94 & 2.29 & 1.48 & 0.64 \\
\hline G2 & 1.07 & 11.29 & 1.93 & 1.16 & 11.19 & 1.66 & 1.10 & 0.66 & 1.08 & 11.36 & 1.87 & 1.23 & 0.65 \\
\hline GM1 & 0.86 & 11.86 & 2.33 & 0.92 & 11.89 & 2.02 & 1.32 & 0.65 & 0.88 & 12.05 & 2.25 & 1.37 & 0.60 \\
\hline TM1 & 0.85 & 12.39 & 2.15 & 0.90 & 12.38 & 1.87 & 1.16 & 0.62 & 0.85 & 12.58 & 2.10 & 1.24 & 0.59 \\
\hline NL3 & 0.67 & 13.19 & 2.74 & 0.76 & 12.83 & 2.32 & 1.62 & 0.70 & 0.69 & 13.35 & 2.65 & 1.73 & 0.65 \\
\hline
\end{tabular}




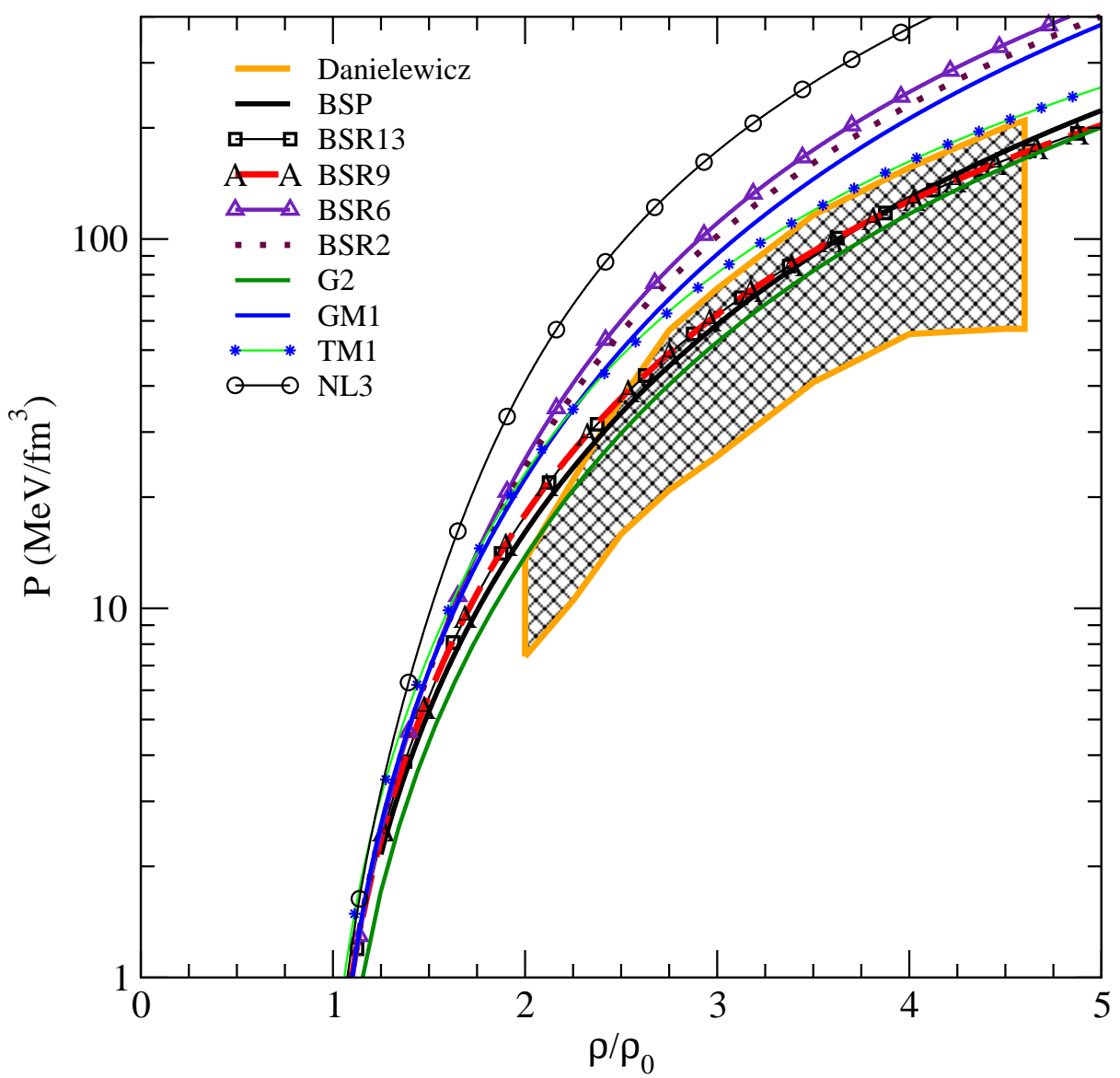

FIG. 1: (Color online) Pressure as a function of nucleon density for the symmetric nuclear matter. The shaded area represents the EOS extracted from the analysis of Ref. [19]. The density is scaled by $\rho_{0}=0.16 \mathrm{fm}^{-3}$. 


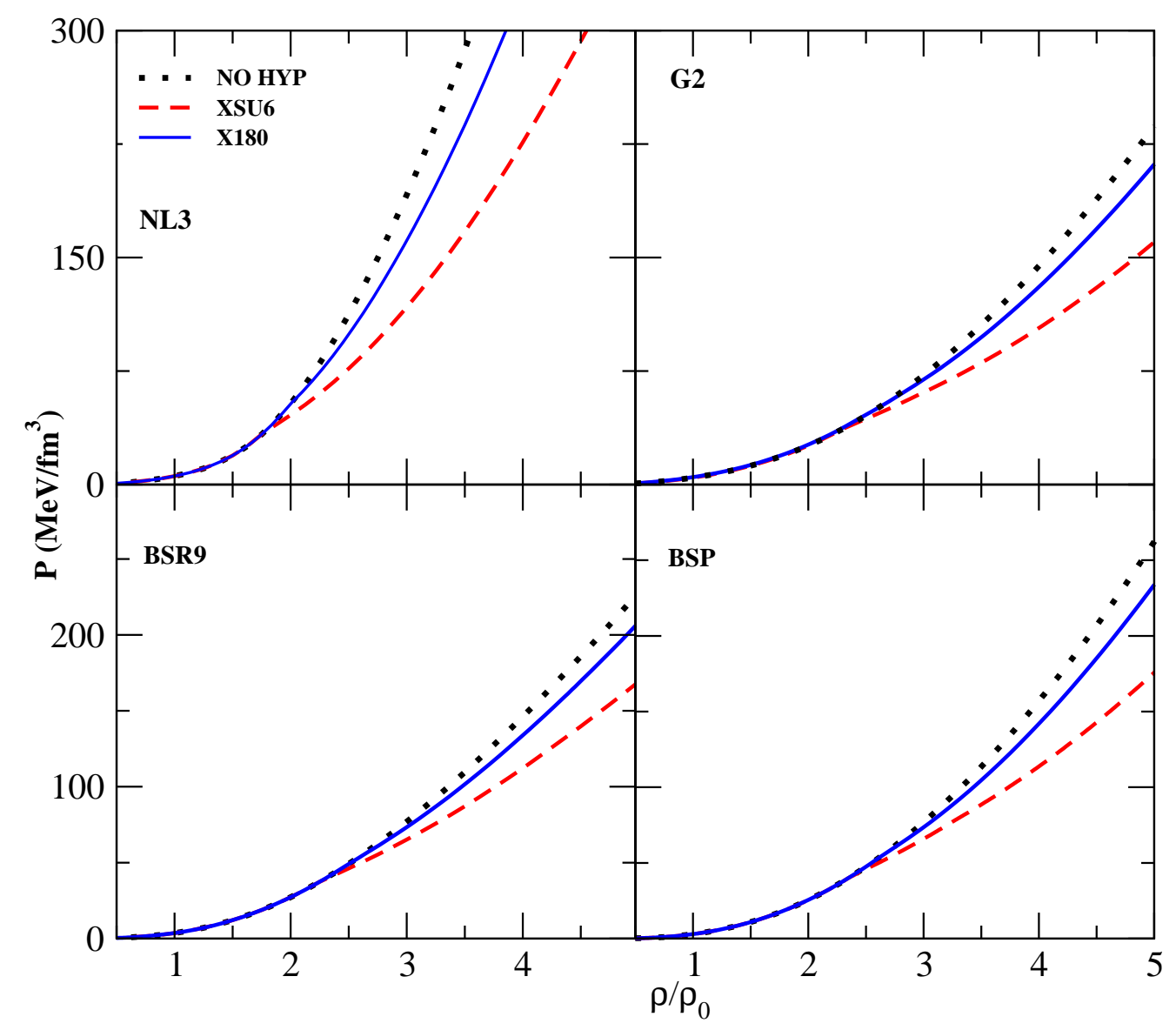

FIG. 2: (Color online) The EOSs in terms of pressure versus density for few RMF models. The curves labeled 'NO HYP' correspond to the EOSs for the nucleonic matter. The EOSs for the hyperonic matter for two different choices for the meson-hyperon coupling strengths are labeled by XSU6 and X180 (see text for detail). 


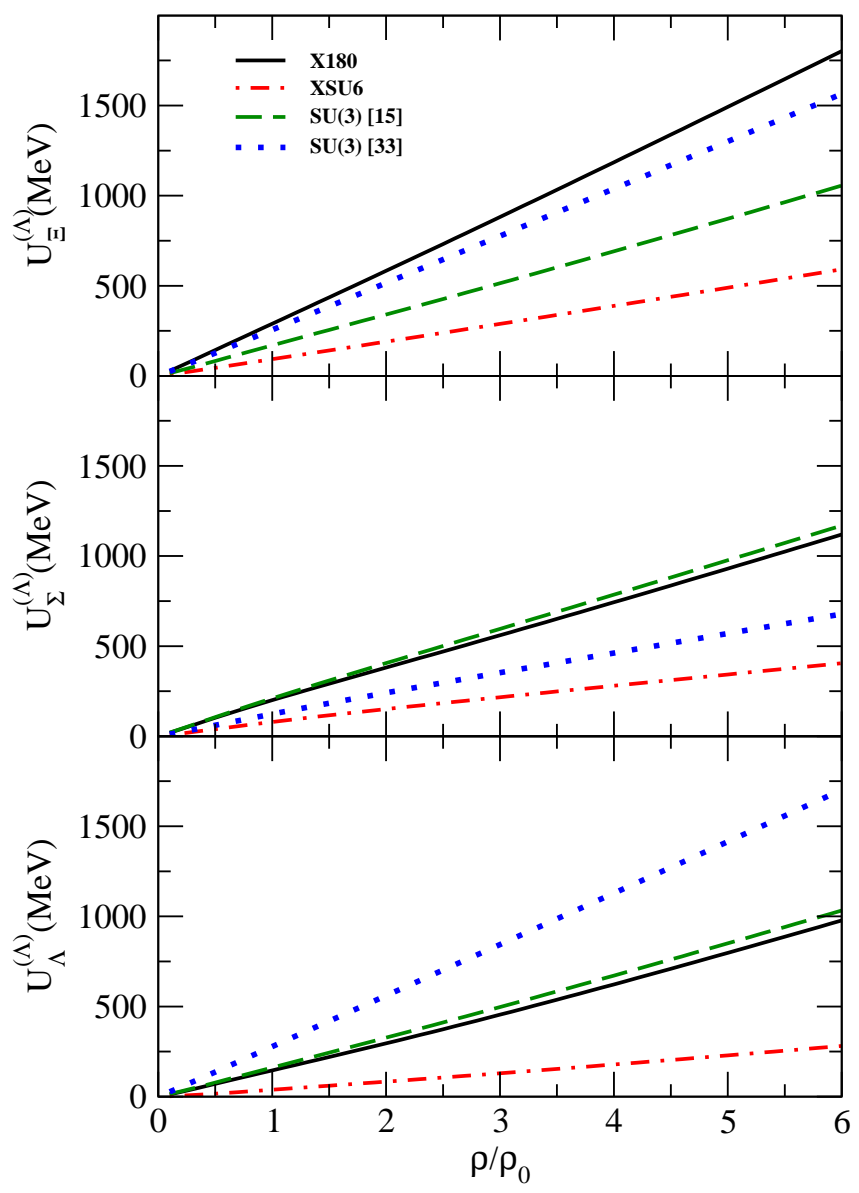

FIG. 3: (Color online) The $\Lambda-\mathrm{H}$ potentials $U_{H}^{(\Lambda)}$ as a function of density for the meson-hyperon coupling strengths corresponding to XSU6 and X180. For the comparison, the values of $U_{H}^{(\Lambda)}$ obtained from SU(3) models [15, 33] are also plotted. 


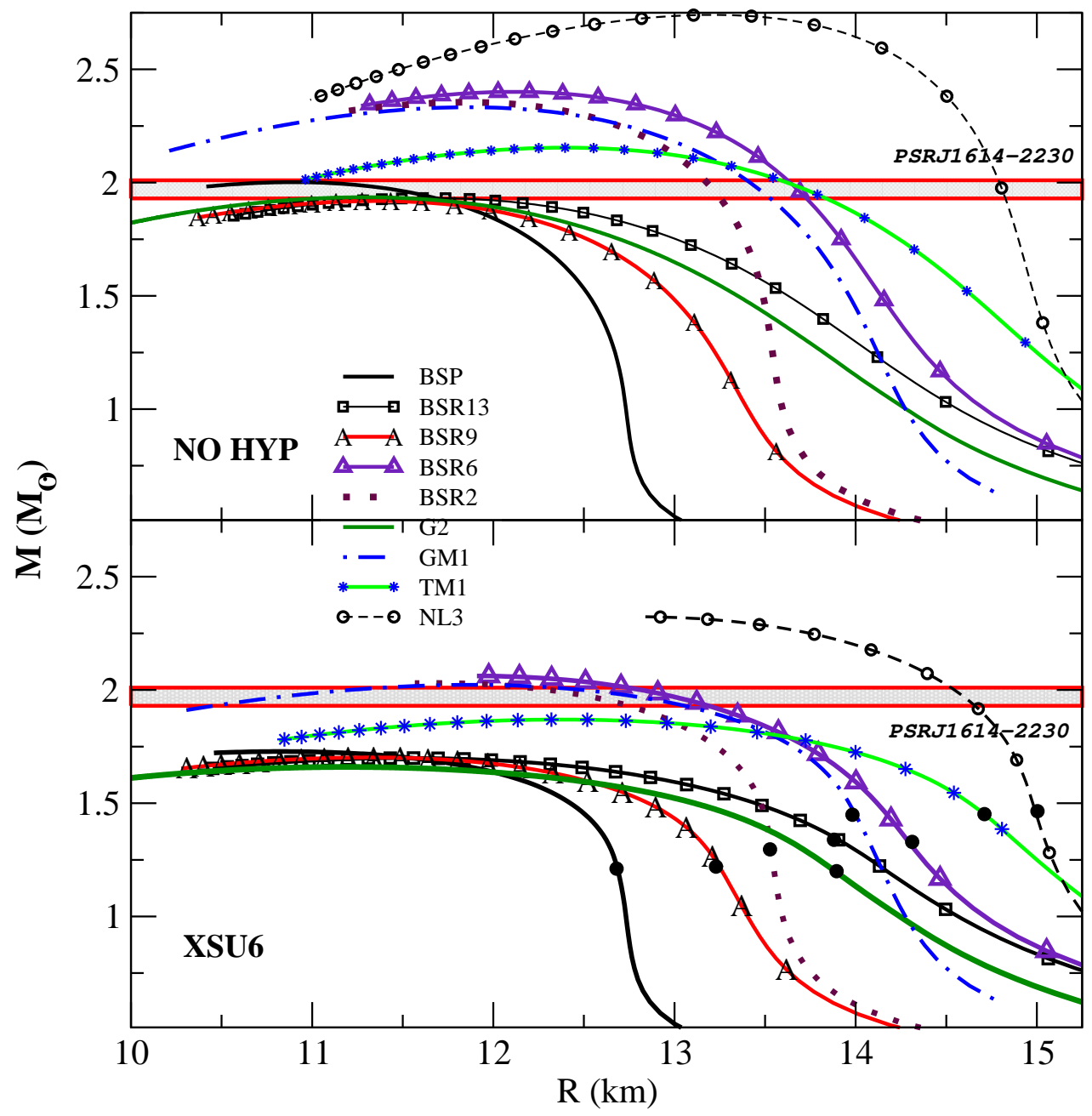

FIG. 4: (Color online) Plots for the mass-radius relationships for the equilibrium sequences of static compact stars obtained using various EOSs for RMF models. The results with no hyperons are depicted in the upper panel and those with hyperons in the lower panel. The label XSU6 in the lower panel represents that the hyperons are included using standard values for the meson-hyperon coupling strengths. The curves on the left of the solid circles represent the equilibrium sequences for the compact stars with core composed of tire hyperonic matter. 


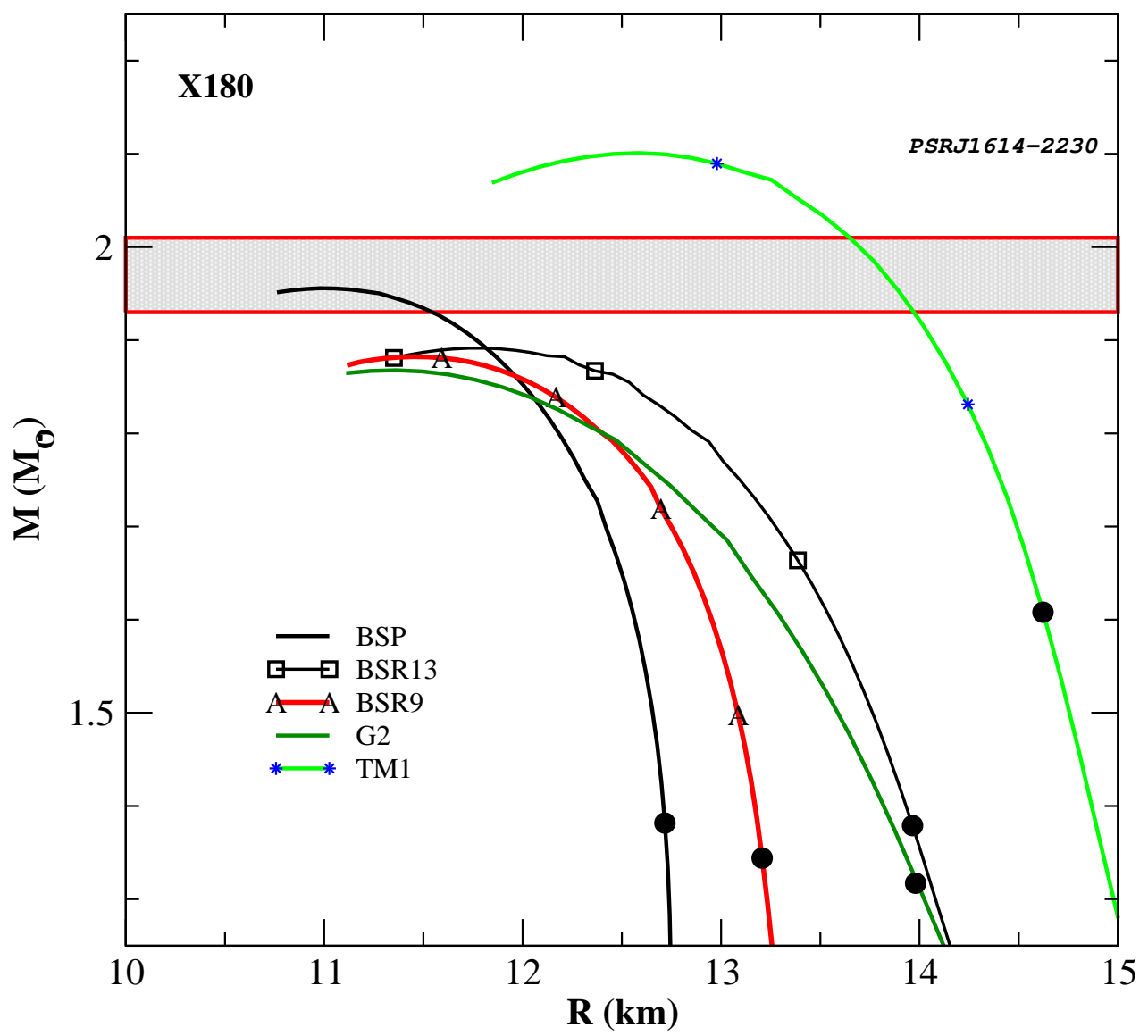

FIG. 5: (Color online) The mass-radius relationships for the static compact stars obtained for the values of the meson-hyperon coupling strengths corresponding to X180. The curves on the left of the solid circles represent the sequences for the compact stars with core composed of the hyperonic matter. 


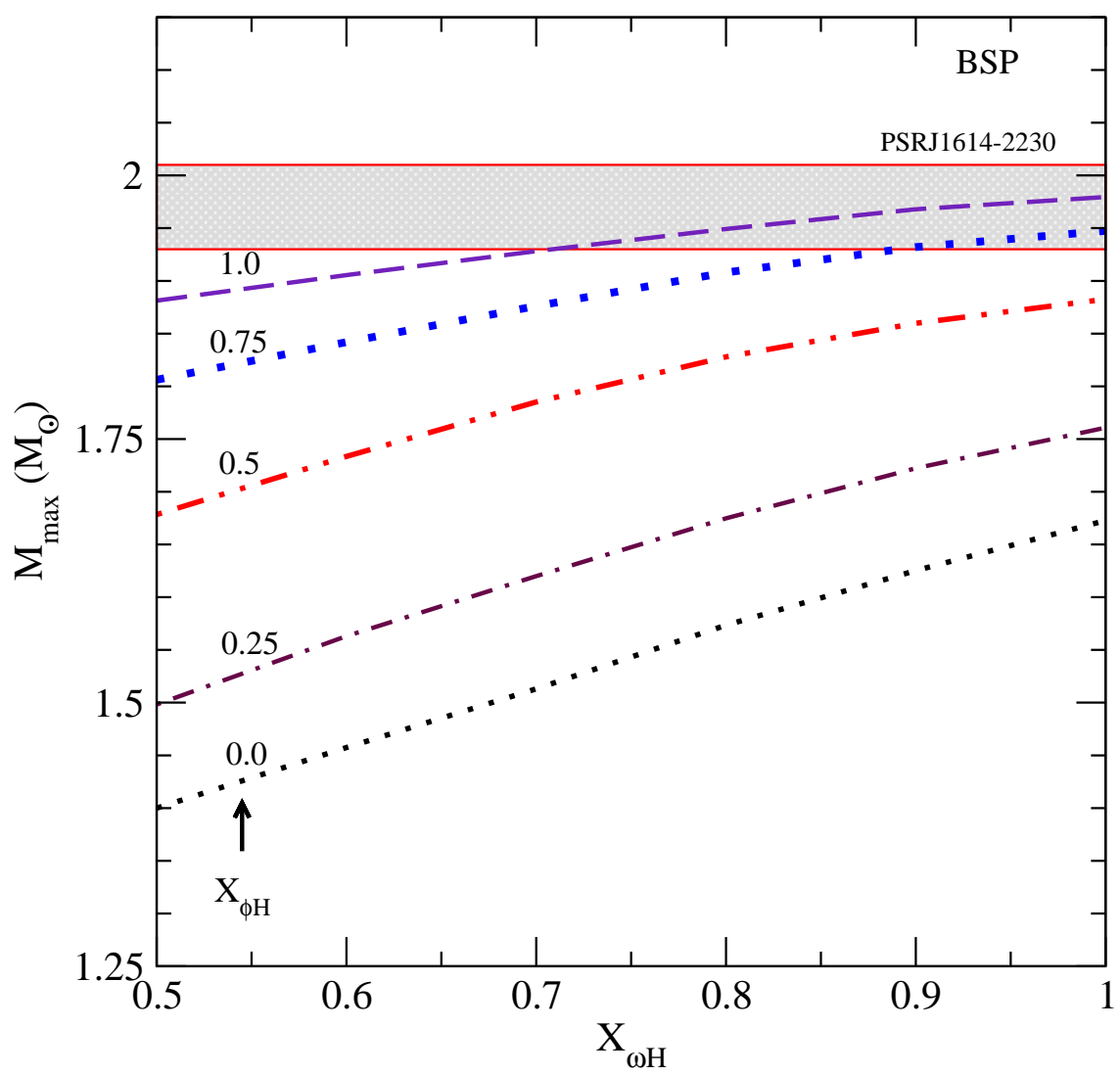

FIG. 6: (Color online) The dependence of the maximum mass $M_{\max }$ for the static compact stars on the values of meson-hyperon coupling strengths $X_{\omega H}$ and $X_{\phi H}$ (Eqs. (10) and (11)) for the case of BSP. 


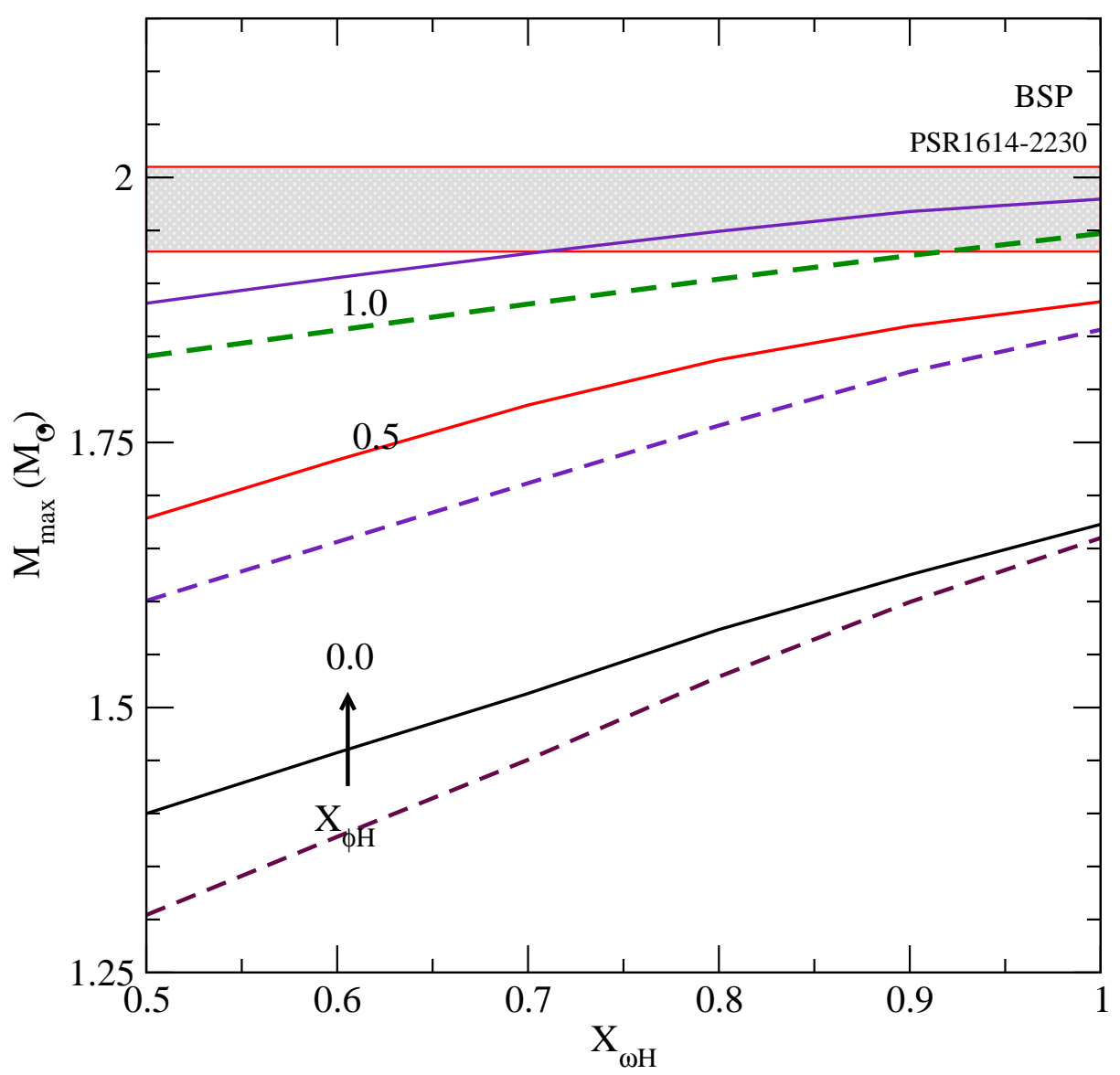

FIG. 7: (Color online) Similar to Fig. 6, But, the values of $M_{\max }$ obtained for $U_{\Sigma}^{(N)}=30 \mathrm{MeV}$ (solid line) are compared with those for $U_{\Sigma}^{(N)}=-30 \mathrm{MeV}$ (dashed line). 


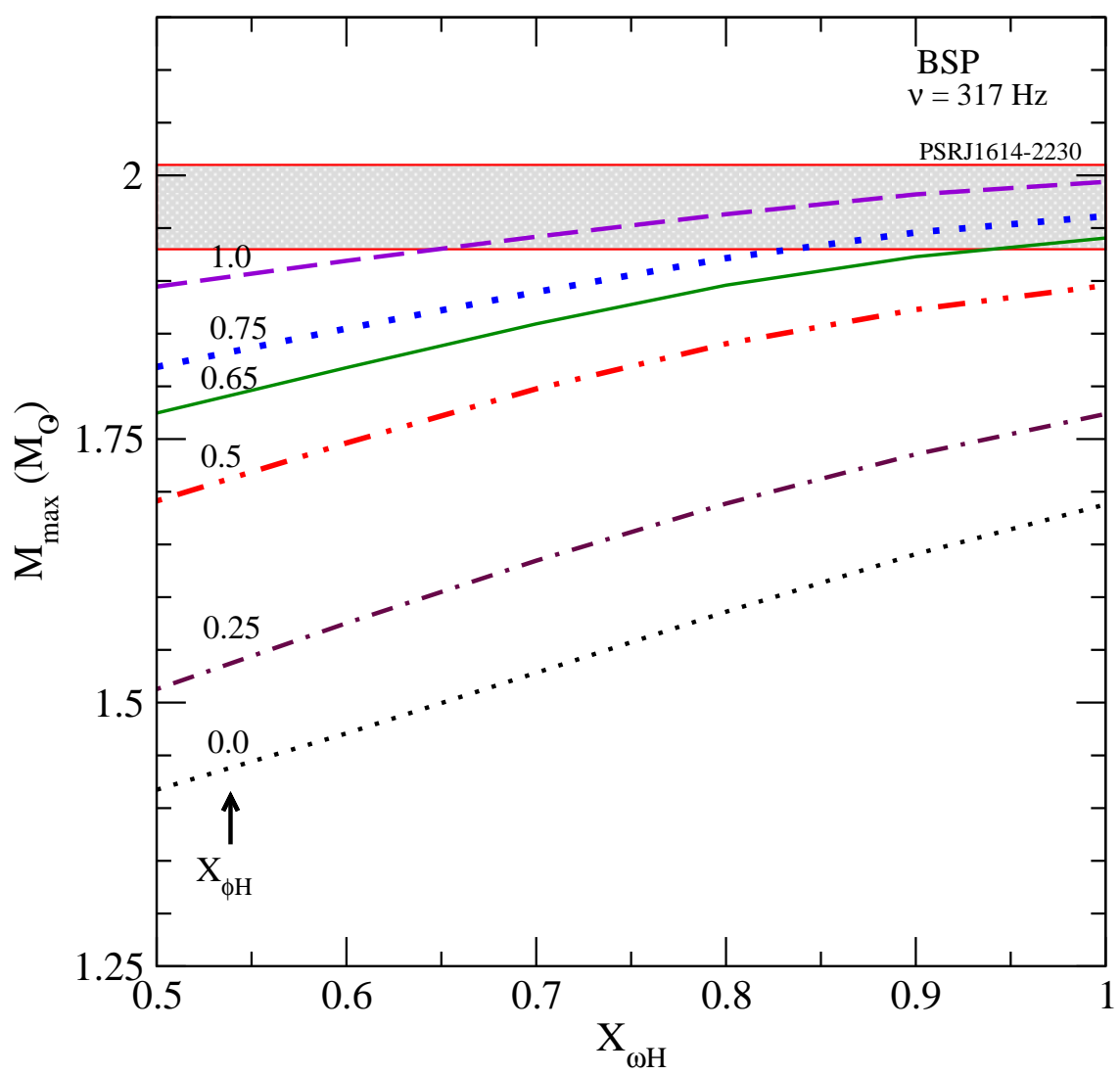

FIG. 8: (Color online) Similar to Fig. 6, But, for the compact stars rotating with the frequency of $317 \mathrm{~Hz}$, same as that of the PSRJ1614-2230. 